\title{
Using the mycelium-covered cereals as an efficient inoculation method for rapamycin fermentation in a 15-L fermenter using Streptomyces hygroscopicus
}

Hong-Wei Yen ${ }^{*}$ and Meng-Hua Chiang

\begin{abstract}
Background: Rapamycin is produced from Streptomyces hygroscopicus, and was initially identified as an antifungal antibiotic. More recently, rapamycin has been found to have various medical applications, including in relation to immunosuppression and anti-aging. Due to its complex structure, biological production is the major route for commercialized rapamycin production. The conventional fermentation process requires a large seed fermenter for the inoculation process (in general, the volume of the seed fermenter is equal to 5-10 \% that of the production fermenter), which presents challenges with regard to scaling up production, due to the high investment costs of seed fermenters. This study explored different inoculation strategies for rapamycin production in a 15-L agitation fermenter.

Results: The results indicated that solid-state fermentation (SSF) using barley as the substrate is a suitable method for the inoculation. The highest rapamycin concentration measured in the batch with SSF (barley) inoculated was about $520 \mathrm{mg} / \mathrm{L}$, which was significantly higher than that of $400 \mathrm{mg} / \mathrm{L}$ obtained in the batch inoculated with $5 \%$ liquid seed medium. Besides the higher rapamycin production, using SSF of barley as the inoculation method can greatly reduce both the labor and cost requirements.

Conclusions: The usage of mycelium-covered barley as the solid substrate for the inoculation of 15-L fermenter leading to a higher rapamycin production compared to that of conventional liquid seed medium. The solid-state inoculation method can avoid both the intensive labor requirement and costly seed fermenter needed with the latter approach. This inoculation method thus has the potential to be applied to the large-scale production of rapamycin.
\end{abstract}

Keywords: Inoculation, Morphology, Solid-state fermentation, Rapamycin

\section{Background}

Rapamycin was initially identified as having the medical function of an antifungal antibiotic, and was purified from an isolated Streptomyces hygroscopicus from Easter island soil in 1975 (Sehgal et al. 1975). Furthermore, rapamycin has been shown to have various medical applications, such as immunosuppression by inhibiting $\mathrm{T}$ cell activation and proliferation (Michaela Kuhnt et al. 1997). Of perhaps more interest is that rapamycin has been

\footnotetext{
*Correspondence: hwyen@thu.edu.tw

Department of Chemical and Materials Engineering, Tunghai University, 1727, Sec. 4, Taiwan Boulevard, Taichung 40704, Taiwan
}

suggested to have a potent anti-aging function, by inhibiting mammalian targeting of rapamycin (mTOR). Due to its wide medical applications, the production of rapamycin through the biological process of S. hygroscopicus cultivation has attracted much attention in recent years.

Various fermentation strategies of S. hygroscopicus for the enhancement of rapamycin production have been explored, such as the optimum carbon source (Kojima et al. 1995) and nitrogen source screening (Lee et al. 1997). Besides the study of medium components, the use of a high dissolved oxygen (DO) environment is beneficial to rapamycin production. Nevertheless, the aim of high rapamycin production is to keep the intact pellet form 
of S. hygroscopicus, even under the high DO conditions. Therefore, the addition of pure oxygen to avoid the high shear force resulting from rapid agitation speed has been carried out, with $780 \mathrm{mg} / \mathrm{L}$ of rapamycin being obtained in a 5-L fermentor batch with DO controlled to over $30 \%$ using pure oxygen (Yen and Hsiao 2013). In addition to $\mathrm{DO}$, it has also been suggested that the $\mathrm{pH}$ value can alter the related metabolic pathway, thus changing the process of rapamycin production. More recently, a two-stage $\mathrm{pH}$ control strategy with no $\mathrm{pH}$ control in the first stage and with the $\mathrm{pH}$ controlled at 5.5 in the second stage was proposed to enhance rapamycin production (Yen et al. 2013).

Besides the effects of fermentation process parameters on rapamycin production, the morphologies of microorganisms also play a crucial role in the resulting metabolite products (Dobson et al. 2008; Ilić et al. 2008; Treskatis et al. 1997). It is well known that the cultivation conditions, including chemical and physical parameters, would change the morphology of actinomyces, and further affect their antibiotic productivity (Chen et al. 1999; Dobson et al. 2008; El-Sabbagh et al. 2006; Kanda et al. 2010). For example, a relationship between morphology and productivity of antibiotics by Streptomyces has been found, including with regard to the composition, structure, hydrophobicity or charge of the cell wall, as well as the presence of extracellular polymeric substances (Choi et al. 1998; Jonsbu et al. 2002; Pinto et al. 2004; Tamura et al. 1997). The morphology of Streptomyces may vary from a free filamentous suspension to pellets, depending on the degree of aggregation. The entanglement of prolonged filaments leads to the formation of pellets in submerged fermentations as a result of the aggregation of spores and/or hyphae. It was reported that such pellet-formed processes depend strongly on the species and the process conditions employed, and there may be a direct or indirect relationship with regard to the effects of morphology on the formation of metabolite products. Morphological control has been achieved by increasing the inoculum size, changing the initial $\mathrm{pH}$ value of the medium, and using additives, such as carboxymethylcellulose (CMC), chelators, Tween-80, or agar granules. For example, a study reported that adding CMC increased the viscosity of the medium and reduced pellet size, thus increasing rapamycin production in the cultivation of $S$. hygroscopicus (Yen and Li 2014). Similarly, smaller pellets can be beneficial to lovastatin production in the cultivation of Aspergillus terreus (Casas Lopez et al. 2005). The inoculation size can also affect the morphology by providing more growing points, due to the high initial cell density. A paper on the cultivation of Ganoderma lucidum found that a large inoculation density led to a small pellet size and high production of extracellular and intracellular polysaccharides (Fang et al. 2002). A review of the literature thus suggests that the production of rapamycin by S. hygroscopicus could be enhanced by the use of a more suitable inoculation method.

Moreover, a suitable inoculation method not only can enhance rapamycin production, but also simplify the commercial fermentation process. In a scaled-up fermentation process a high inoculation density means a long inoculation time is required, which increases costs and reduces the economic feasibility of such an approach. Therefore, this study aimed to examine the effects of inoculation strategy on rapamycin production, including the inoculation ratio, amount of spores and type of seeding. The aim is to find a suitable inoculation strategy for rapamycin production in a scaled-up fermentation process that is both efficient and convenient.

\section{Methods}

\section{Strain and medium}

The strain used in the experiment, Streptomyces hygroscopicus, was purchased from the Bioresource Collection and Research Center, Taiwan, under the catalog number BCRC 16270 (the same as ATCC 29253). A spore suspension was used for the inoculation of S. hygroscopicus cultivation in this study. The spore suspension aliquot was prepared according to the following procedure; agar plates with the sporulation medium $(4 \mathrm{~g} / \mathrm{L}$ dextrose, $20 \mathrm{~g} / \mathrm{L}$ agar, $4 \mathrm{~g} / \mathrm{L}$ yeast extract and $10 \mathrm{~g} / \mathrm{L}$ malt extract) were plated out with the prepared spore solution and incubated at $28{ }^{\circ} \mathrm{C}$ for $20-25$ days. After the black spores were seen to spread on the surface of agar plates, $1 \mathrm{~mL}$ of sterilized water was added to the plates to harvest the spore suspension, and preserved in frozen stock (stored in $25 \%$ glycerin at $-20{ }^{\circ} \mathrm{C}$ ). The count of the prepared spore suspension was about $10^{8}$ spores $/ \mathrm{mL}$, as measured in the colony-forming unit.

\section{Inoculation methods}

Three inoculation methods were performed in this study, including liquid seed medium inoculation, spore suspension inoculation and mycelium inoculation via solid-state fermentation. Liquid seed medium inoculation was performed using conventional liquid seed medium preparation procedure. A seed culture was initiated by adding $1 \mathrm{~mL}$ of the thawed cell suspension to a 250-mL Erlenmeyer flask containing $100 \mathrm{~mL}$ of seed medium $(4 \mathrm{~g} / \mathrm{L}$ dextrose, $4 \mathrm{~g} / \mathrm{L}$ yeast extract, $10 \mathrm{~g} / \mathrm{L}$ malt extract) at the initial pH 7.3 for $48 \mathrm{~h}$ (Yen and Hsiao 2013). A defined inoculation ratio of 2,5 and $10 \%(\mathrm{v} / \mathrm{v})$ of seed medium was then inoculated into a $15-\mathrm{L}$ agitation fermentor. The spore suspension was prepared in the petri-dishes containing the solid seed medium, as described above. After 20-25 days of cultivation, the black spores were observed on the surface of agar plate. Sterile water was used to 
collect the spores from the surface for the preparation of spore suspension. After the spore suspension was collected, it was inoculated into the 15-L fermentor directly, without the seed medium stage. Two concentrations of spore suspension were examined in this study, $1 \times$ $10^{10} / \mathrm{mL}$ (denoted as the low SS batch) and $2 \times 10^{10} / \mathrm{mL}$ (denoted as the high SS batch). The batch with mycelium inoculation of cereals cultured by solid-state fermentation (SSF) was performed as following: a seed culture was initiated by adding $1 \mathrm{~mL}$ of the thawed cell suspension to a 250-mL Erlenmeyer flask containing $100 \mathrm{~mL}$ of seed medium $(4 \mathrm{~g} / \mathrm{L}$ dextrose, $4 \mathrm{~g} / \mathrm{L}$ yeast extract, $10 \mathrm{~g} / \mathrm{L}$ malt extract) (Yen and Hsiao 2013) at the initial $\mathrm{pH} 7.3$ for $48 \mathrm{~h}$. A $1 \mathrm{~mL}$ aliquot of the seed culture was then transferred into a solid medium containing $10 \mathrm{~g}$ of cereal or bean and $10 \mathrm{~mL}$ of water, incubated at $28{ }^{\circ} \mathrm{C}$ for 10 days. Eight crops that often be investigated as substrates for solid fermentation, namely barley, buckwheat, brown rice, polished rice, red beans, soybean and green beans (mung bean) were examined in this study (Yang et al. 2013). After 10 days of cultivation, the white mycelium covered the cereals, and the whole $10 \mathrm{~g}$ of each crops along with the mycelium was liquefied with some sterile water using a homogenizer. The homogenized mycelium solution (with the cereals or beans) was then inoculated into the $15-\mathrm{L}$ fermentor.

\section{The fermentation in a 15-L agitation fermentor}

The steps used to prepare the seed cultivation and medium components were the same as described in the previous section, except for the addition of $0.05 \%$ antifoaming agent in the fermentation medium, which prevented foaming under severe agitation.

A seed medium was prepared and inoculated into a $15-\mathrm{L}$ fermentor containing a $10-\mathrm{L}$ working volume $(4 \mathrm{~g} / \mathrm{L}$ of yeast extract, $10 \mathrm{~g} / \mathrm{L}$ of malt extract and $25 \mathrm{~g} / \mathrm{L}$ of glucose). The fermentor was maintained at $28{ }^{\circ} \mathrm{C}$ and an aeration rate of $1 \mathrm{vvm}$ was adopted. The DO level was controlled at about $30 \%$ by adjusting the agitation rate automatically. However, the maximum agitation rate was limited to be no higher than $200 \mathrm{rpm}$ to avoid damaging the pellets.

\section{Analytical methods}

The glucose concentration was estimated using a glucose analysis instrument assay (YSI 2300 STAT Glucose Analyzer). Dry biomass was estimated using the moisture analyzer (IR-35, DENVER) assay; $5 \mathrm{~mL}$ of whole broth was centrifuged at $7000 \mathrm{rpm}$ for $5 \mathrm{~min}$ to harvest the precipitated biomass. Following this, $1 \mathrm{~mL}$ of purified water was added into the tube to complete the aqueous mixing of the biomass. The whole aqueous biomass was transferred onto the IR dryer, and water was evaporated by infrared radiation to estimate the proportion of dry biomass. The measurement of rapamycin titer was performed by the HPLC method. A 1-mL aliquot of fermentation broth was centrifuged at $7000 \mathrm{rpm}$ for $5 \mathrm{~min}$. The supernatant was carefully transferred by pipette into a test tube, and the precipitate pellet was extracted again by shaking it with $1 \mathrm{~mL}$ of methanol for $20 \mathrm{~min}$ at room temperature. The extractant was subjected to HPLC analysis (Hitachi), using a Vercopak C18 column under the following conditions: mobile phase:methanol/water/acetic acid, 80/20/0.1; flow rate: $1.2 \mathrm{~mL} / \mathrm{min}$; detector: UV $254 \mathrm{~nm}$ (this method was suggested by LC Laboratories) (Yen et al. 2013). The standard rapamycin was purchased from LC Laboratories, USA (catalog number 53123-88-9, purity $>99 \%$ ).

\section{Results and discussion}

\section{Effects of inoculation ratio of liquid seed in a 15-L agitation} fermentor

The morphology of microorganisms is known to be affected by the inoculation ratio, and the morphology can further change the metabolic pathway and the subsequent production of metabolites (Fang et al. 2002). Therefore, the effects of inoculation ratio on rapamycin production were examined in this study. Three ratios of 2,5 and $10 \%(\mathrm{v} / \mathrm{v})$ were used in a $15-\mathrm{L}$ agitation bioreactor for the comparison of rapamycin production, and the results are shown in Fig. 1. No significant differences in the morphology were observed among all batches with different inoculation ratios, as all were in loose pellet form, indicating that the inoculation ratio would not affect cell coagulation in the agitation tank. The size of the loose pellets was about $0.5-1 \mathrm{~cm}$, and they had an irregular appearance. From the previous study, it was known that several fermentation conditions (such as dissolved oxygen level and medium viscosity) would affect the pellet morphology (Yen and Hsiao 2013). Due to the effects of high shear force resulting from the agitation, a lot of mycelium were observed in the broth among all batches with various inoculation ratios. The similar morphology led to the similar biomass that was obtained for all batches. As for the rapamycin production, the batches with 10 and $5 \%$ inoculation ratios had similar rapamycin concentrations, which were higher than that obtained with the $2 \%$ batch. Besides the rapamycin concentration, the batch with a $2 \%$ inoculation ratio had the lowest rapamycin content of $26.7 \mathrm{mg} / \mathrm{g}$, as compared to 51 and $60 \mathrm{mg} / \mathrm{g}$ in the batches with 5 and $10 \%$, respectively. The results thus suggest that the inoculation ratio would not significantly change the morphology, although it did affect the production of rapamycin. An inoculation ratio of at least $5 \%$ is thus suggested to achieve relatively good rapamycin production. 


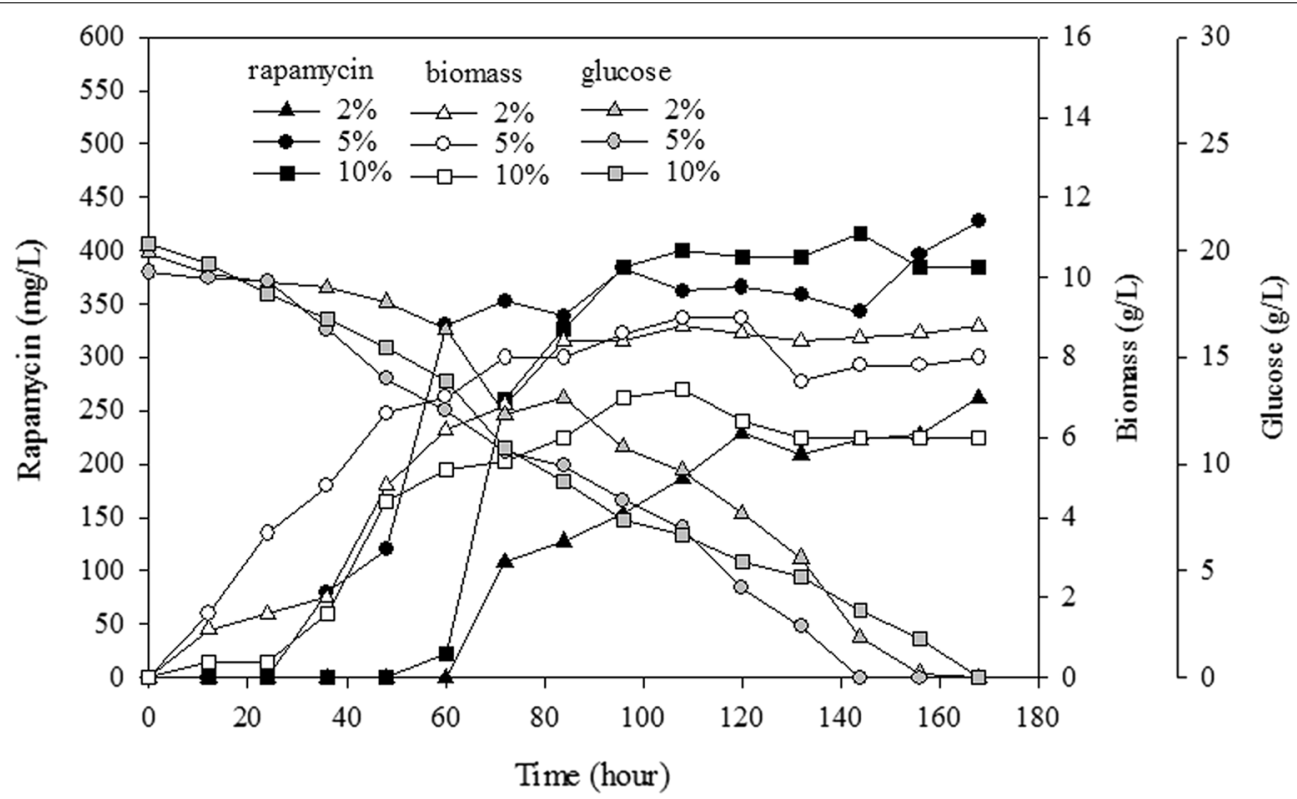

Fig. 1 Effects of liquid seed medium inoculation at the ratios of 2, 5 and $10 \%(\mathrm{~V} / \mathrm{V})$ on rapamycin production in a 15-L fermentor with DO controlled at about $30 \%$ and at $28^{\circ} \mathrm{C}$

\section{Using spore suspension for the inoculation}

As noted in the previous discussion, an inoculation ratio of least $5 \%$ is suggested to achieve good rapamycin production. However, a higher inoculation ratio means that a larger seed tank is needed to satisfy the requirement of a $5 \%$ inoculation ratio, and this might present some problems with regard to scaling up the process for industrial fermentation. The performance when directly using prepared spore suspension for the inoculation, instead of liquid seed medium, was thus examined here. The spore suspension was collected from the mycelium-covered agar plate using sterilized water to have the black spores harvested. A spore suspension of $100 \mathrm{ml}$ with spore concentrations of $1 \times 10^{10} / \mathrm{mL}$ (low SS) and $2 \times 10^{10} / \mathrm{mL}$ (high SS) were thus inoculated into the fermentor in two separate experiments. The results for the low and high SS batches are shown in Fig. 2, and compared to those for the batch with $5 \%$ of liquid seed medium. It can be seen that the batch inoculated with $5 \%$ of liquid seed medium led more rapamycin being produced than when the spore suspensions were used, although the latter produced similar amounts of biomass. In addition to the lower rapamycin production, the batches directly inoculated with spore suspension also had a longer lag phase. The results thus indicated that inoculation using a spore suspension is not a suitable strategy for producing rapamycin on an industrial scale.

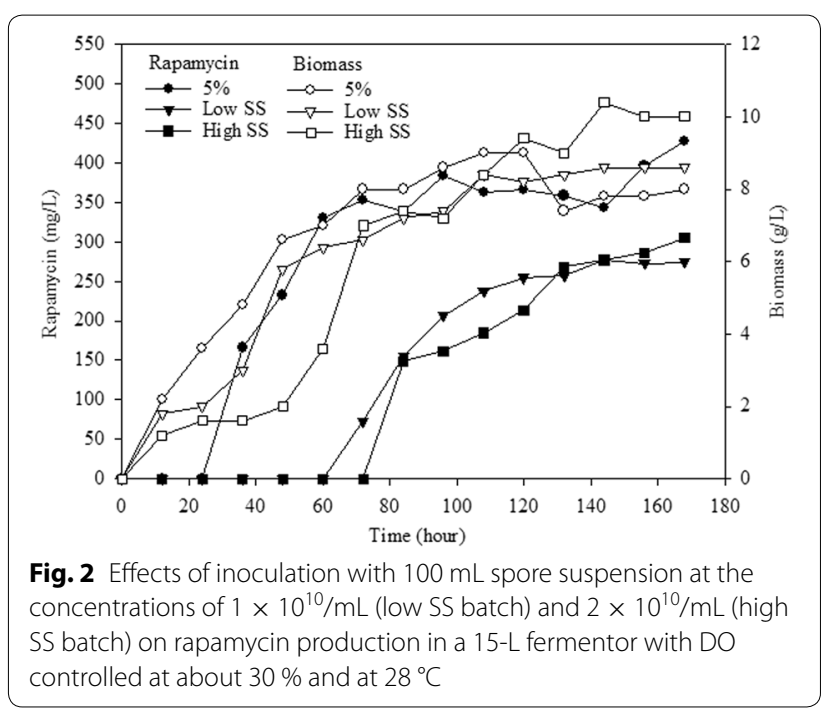

The inoculation using mycelia-covered cereals

As noted in the previous section, direct inoculation with a spore suspension is not a good method for the largescale rapamycin production, and thus solid-state fermentation (SSF) of cereals was examined as the inoculation method instead of conventional liquid seed culture. Several cereals and beans were first tested to use as the substrate for the growth of S. hygroscopicus in SSF, namely 
barley, buckwheat, brown rice, glutinous rice, red bean, soybean and green bean (mung bean). The results in Fig. 3 show that barley is the best substrate for the growth of S. hygroscopicus among all the investigated crops, based on the resulting rapamycin production, while mung bean is the worst. Indeed, none of the bean crops (red, soy and mung beans) were good substrates for the growth of S. hygroscopicus. After 10 days of cultivation, white mycelium was appeared on the surface of all the cereals and beans. However, the mycelium covered the entire surface of the batch using barley as the

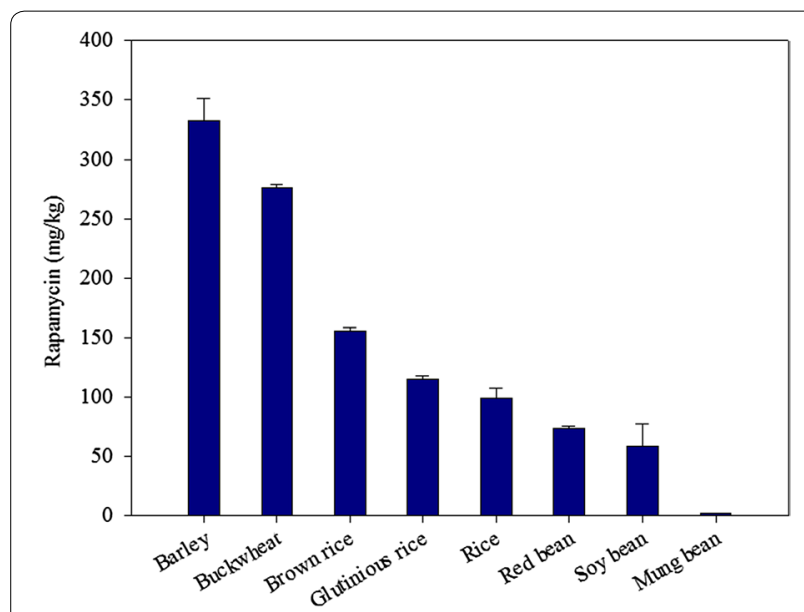

Fig. 3 Rapamycin production using solid-state fermentation (SSF) and various crops at $10 \mathrm{~g}$ each with $10 \mathrm{~mL}$ of water, 10 days of cultivation at $28^{\circ} \mathrm{C}$ substrate, and this also had the highest rapamycin production per $\mathrm{kg}$ of crop used. It is thus recommended that barley could be used as the substrate for this submerged fermentation process. The differences in the protein or carbohydrate contents or the $\mathrm{C} / \mathrm{N}$ ratios of the various substrates might be speculated as the reason leading to the increase of rapamycin using barley (Yang et al. 2013). In addition to the potential nutrients effects, the physical properties, particularly the smaller particle size of barley make it have the more availability of the nutrients, which could also be the reason leading to the superiority of the barley over the other solid substrates on rapamycin production (Yang et al. 2013). However, further works might be needed to elucidate the reasons in detail.

When using mycelium-covered barley as the inoculation method, the whole barley solids were first homogenized with sterilized water to obtain a homogenous solution, and then inoculated into a $15-\mathrm{L}$ agitation fermentor. Figure 4 shows the results obtained with the barley-inoculated batch as compared to the conventional inoculation approach with $5 \%$ liquid seed medium. These show that the production of rapamycin in the batch inoculated with barley had a longer lag phase than that inoculated with the $5 \%$ liquid seed medium. Nevertheless, the final rapamycin concentration of the barely inoculated batch was higher than that obtained with the conventional $5 \%$ liquid seed medium. Since the solid barley substrate was inoculated, the biomass measurements were not very precise at the beginning of the fermentation process, and thus a higher biomass

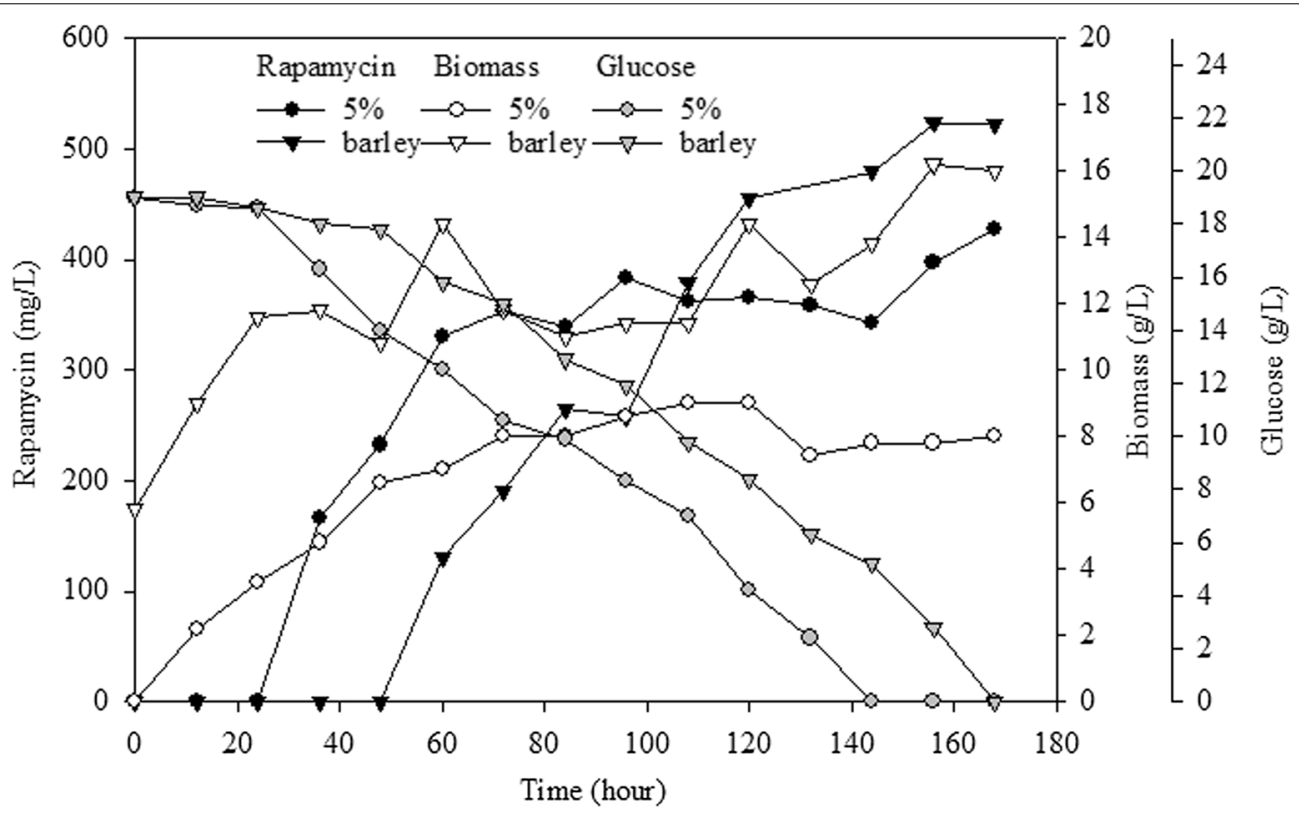

Fig. 4 Effects of inoculation with mycelia-covered barley through SSF cultivation on rapamycin production in a 15-L fermentor as compared to the conventional liquid seed medium inoculated at $5 \%$ of ratio 
was observed. Nevertheless, the results suggest that inoculation with mycelium-covered barley cultured by SSF could have a relatively higher cell growth rate than seen with the control batch inoculated with $5 \%$ liquid medium, as well as a higher rapamycin production. The highest rapamycin concentration that was measured in the batch with inoculated with barley was about $520 \mathrm{mg} / \mathrm{L}$, significantly higher than that of $400 \mathrm{mg} / \mathrm{L}$ obtained in the batch inoculated with $5 \%$ liquid seed medium. In addition to the higher rapamycin production, the preparation of mycelium-covered barley solids would avoid the need for a larger seed fermentor when scaling up rapamycin production, even though the solid-state cultivation of S. hygroscopicus required a longer period (10 days in this study) as compared to the 1-2 days required with submerged seed cultivation. The ease of preparation, lower cost process and no need for a large seed fermentor thus make solid-state fermentation very attractive as the inoculation method when compared to the conventional liquid medium approach.

A comparison of several parameters among various inoculation criteria is shown in Table 1 with regard to the liquid seed medium, spore suspension and myceliumcovered barley approaches. It can be seen that the batch with mycelium-covered barley had the highest rapamycin production of $520 \mathrm{mg} / \mathrm{L}$, and a relatively high rapamycin productivity of $3.6 \mathrm{mg} / \mathrm{L} \mathrm{h}$. Even though conventional liquid seed inoculation at $10 \%$ had a slightly higher rapamycin productivity of $3.7 \mathrm{mg} / \mathrm{L} \mathrm{h}$, a larger seed tank required at a higher inoculation ratio would cause problems with regard to commercial production, due to the greater capital requirement for a more costly fermentor. Therefore, the use of solid barley cultured in SSF for inoculation is more appropriate than that of the conventional liquid seed medium, as it can greatly reduce the cost of this process, and thus make it more economically viable.

The most important thing for an ideal inoculation method is trying to avoid the use of costly seed fermentor in the commercialized rapamycin production. The results indicated that solid-state fermentation (SSF) using barley as the substrate is a suitable method for inoculation. This led to higher rapamycin production compared to that seen with $5 \%$ liquid seed medium, and avoided both the intensive labor requirement and costly seed fermentor needed with the latter approach. The comparison of kinetic parameters obtained in the batch using barley as the seed inoculation with data in literature is shown in Table 2.

Table 1 The comparison of rapamycin production parameters between batches with various inoculation criteria into a 15-L fermenter: liquid seed medium at the ratios of 2, 5 and $10 \%(v / v)$, spore suspension at low and high spore concentrations and mycelium-covered barley inoculated

\begin{tabular}{|c|c|c|c|c|}
\hline $\begin{array}{l}\text { Inoculation } \\
\text { methods }\end{array}$ & $\begin{array}{l}\text { Max. biomass } \\
\text { (g/L) }\end{array}$ & $\begin{array}{l}\text { Max. rapamycin } \\
(\mathrm{mg} / \mathrm{L})\end{array}$ & $\begin{array}{l}\text { Rapamycin } \\
\text { content (mg/g cells) }\end{array}$ & $\begin{array}{l}\text { Rapamycin } \\
\text { productivity (mg/L h) }\end{array}$ \\
\hline \multicolumn{5}{|c|}{ Liquid seed medium ${ }^{\mathrm{a}}$} \\
\hline $2 \%$ & 8.8 & 229 & $26 \pm 3$ & 1.91 \\
\hline $5 \%$ & 9.1 & 400 & $41 \pm 3$ & 3.04 \\
\hline $10 \%$ & 7.2 & 400 & $56 \pm 5$ & 3.71 \\
\hline \multicolumn{5}{|l|}{ Spore suspension ${ }^{\mathrm{b}}$} \\
\hline Low SS & 8.4 & 277 & $33 \pm 2$ & 1.92 \\
\hline High SS & 10.4 & 285 & $28 \pm 3$ & 1.83 \\
\hline Mycelium-barley & 14.4 & 520 & $36 \pm 3$ & 3.61 \\
\hline
\end{tabular}

a Inoculation ratio $=$ (seed medium volume/fermentation volume)

${ }^{b}$ Low $\mathrm{SS}=1 \times 10^{10}$ spore counting per $\mathrm{ml}$ of inoculated solution; high $\mathrm{SS}=2 \times 10^{10}$ spore counting per $\mathrm{ml}$ of inoculated solution

Table 2 A comparison of rapamycin production by using SSF inoculation with other conventional liquid seed fermentations

\begin{tabular}{|c|c|c|c|c|c|}
\hline Carbon source & Bioreactor & Mode & Max. rapamycin (mg/L) & Rapamycin productivity (mg/L h) & References \\
\hline Glucose & $5 \mathrm{~L}$ & Batch & 780 & 4.33 & (Yen and Hsiao 2013) \\
\hline Fructose & $5 \mathrm{~L}$ & Batch & 723 & 6.0 & (Yen and Li 2014) \\
\hline Glycerol, fructose & $130 \mathrm{~L}$ & Fed-batch & 110 & 1.0 & (Chen et al. 1999) \\
\hline Glycerol, fructose & $5 \mathrm{~L}$ & Fed-batch & 500 & 4.17 & (Chen et al. 2008) \\
\hline Dextrin, glycerol & $120 \mathrm{~L}$ & Fed-batch & 812 & 5.08 & (Zhu et al. 2010) \\
\hline Glucose & $15 \mathrm{~L}$ & Batch + SSF & 520 & 3.61 & This study \\
\hline
\end{tabular}




\section{Conclusions}

This study explored different inoculation strategies for rapamycin production in a $15-\mathrm{L}$ agitation fermenter, to reduce the scale-up production costs resulting from the conventional expensive inoculation method. The results indicated that solid-state fermentation (SSF) using barley as the substrate is a suitable method for inoculation. This led to higher rapamycin production compared to that seen with $5 \%$ liquid seed medium, and avoided both the intensive labor requirement and costly seed fermenter needed with the latter approach. This inoculation method thus has the potential to be applied to the largescale production of rapamycin.

\section{Authors' contributions}

$\mathrm{MH}$ carried out the most experiments and analysis. HW conceived of the study, participated in the experimental design and also be responsible for the preparation of this manuscript. Both authors read and approved the final manuscript.

\section{Acknowledgements}

The authors gratefully acknowledge the financial support this study received from Taiwan's Ministry of Science and Technology (MOST) under Grant Number 103-2623-E-029-001-ET and 102-2221-E-029-017-MY2.

\section{Competing interests}

The authors declare that they have no competing interests.

Received: 3 September 2015 Accepted: 4 November 2015

Published online: 17 November 2015

\section{References}

Casas Lopez JL, Sanchez Perez JA, Fernandez Sevilla JM, Rodriguez Porcel EM, Chisti Y (2005) Pellet morphology, culture rheology and lovastatin production in cultures of Aspergillus terreus. J Biotechnol 116:61-77

Chen $Y$ et al (1999) New process control strategy used in a rapamycin fermentation. Process Biochem 34:383-389

Chen Y, Krol J, Huang W, Cino JP, Vyas R, Mirro R et al (2008) DCO2 on-line measurement used in rapamycin fed-batch fermentation process. Process Biochem 43:351-355

Choi DB, Park EY, Okabe M (1998) Improvement of tylosin production from Streptomyces fradiae culture by decreasing the apparent viscosity in an air-lift bioreactor. J Ferment Bioeng 86:413-417

Dobson LF, O'Cleirigh CC, O'Shea DG (2008) The influence of morphology on geldanamycin production in submerged fermentations of Streptomyces hygroscopicus var. geldanus. Appl Microbiol Biotechnol 79:859-866
El-Sabbagh N, McNeil B, Harvey LM (2006) Dissolved carbon dioxide effects on growth, nutrient consumption, penicillin synthesis and morphology in batch cultures of Penicillium chrysogenum. Enzym Microbial Technol 39:185-190

Fang QH, Tang YJ, Zhong JJ (2002) Significance of inoculation density control in production of polysaccharide and ganoderic acid by submerged culture of Ganoderma lucidum. Process Biochem 37:1375-1379

\|lić SB, Konstantinović SS, Veljković VB, Savić DS, Lazić, Gojgić-Cvijović G (2008) Impact of carboxymethylcellulose on morphology and antibiotic production by Streptomyces hygroscopicus. Curr Microbiol 57:8-11

Jonsbu E, Mclntyre M, Nielsen J (2002) The influence of carbon sources and morphology on nystatin production by Streptomyces noursei. J Biotechnol 95:133-144

Kanda M, Yamamoto E, Hayashi A, Yabutani T, Yamashita M, Honda H (2010) Scale-up fermentation of echinocandin type antibiotic FR901379. J Biosci Bioeng 109:138-144

Kojima I, Cheng YR, Mohan V, Demain AL (1995) Carbon source nutrition of rapamycin biosynthesis in Streptomyces hygroscopicus. J Ind Microbiol Biotechnol 14:436-439

Kuhnt Michaela, Bitsch F, Ponelle M, Fehr T, Sanglier J-J (1997) Microbial conversion of rapamycin. Enzym Microbial Technol 21:405-412

Lee M, Kojima I, Demain A (1997) Effect of nitrogen source on biosynthesis of rapamycin by Streptomyces hygroscopicus. J Ind Microbiol Biotechnol 19:83-86

Pinto LS, Vieira LM, Pons MN, Fonseca MM, Menezes JC (2004) Morphology and viability analysis of Streptomyces clavuligerus in industrial cultivation systems. Bioprocess Biosyst Eng 26:177-184

Sehgal SN, Baker H, Vezina C (1975) Rapamycin (AY-22,989), A new antifungal antibiotic II. Fermentation, isolation and characterization. J Antibio 28:727-732

Tamura S, Park Y, Toriyama M, Okabe M (1997) Change of mycelial morphology in tylosin production by batch culture of Streptomyces fradiae under various shear conditions. J Ferment Bioeng 83:523-528

Treskatis SK, Orgeldinger V, Wolf H, Gilles ED (1997) Morphological characterization of filamentous microorganisms in submerged cultures by on-line digital image analysis and pattern recognition. Biotechnol Bioeng 53:191-201

Yang FC, Yang YH, Lu HC (2013) Enhanced antioxidant and antitumor activities of Antrodia cinnamomea cultured with cereal substrates in solid state fermentation. Biochem Eng J 78:108-113

Yen HW, Hsiao HP (2013) Effects of dissolved oxygen level on rapamycin production by pellet-form of Streptomyces hygroscopicus. J Biosci Bioeng 116:366-370

Yen HW, Li YL (2014) The effects of viscosity and aeration rate on rapamycin production in an airlift bioreactor by using Streptomyces hygroscopicus. J Taiwan Inst Chem Eng 45:1149-1153

Yen HW, Hsiao HP, Chen LJ (2013) The enhancement of rapamycin production using Streptomyces hygroscopicus through a simple pH-shifted control. J Taiwan Inst Chem Eng 44:743-747

Zhu X, Zhang W, Chen X, Wu H, Duan Y, Xu Z (2010) Generation of High Rapamycin Producing Strain via Rational Metabolic Pathway-Based Mutagenesis and Further Titer Improvement With Fed-Batch BioprocessOptimization. Biotechnol Bioeng 107:506-515 